\title{
Rapid Mathematical Programming for Cooperative Truck Networks
}

\author{
Jörg Rambau \\ University of Bayreuth, 95440 Bayreuth, Germany, \\ joerg.rambau@uni-bayreuth.de, \\ WWW home page: http://rambau.wm. uni-bayreuth.de
}

\begin{abstract}
We use exact mathematical modeling and optimization to evaluate various operational modes of a cooperative truck network for full-truckload transportation.
\end{abstract}

Keywords: modeling, full-truckload, mixed-integer linear programming

\section{Cooperative Truck Networks}

Cooperative Truck Networks (CTN) are an idea from [1]: In order to reduce idle times of the trucks and overnight stays of the drivers, cooperate among various (small) logistic companies in the following way: Any driver leaves the depot with a truck and a full trailer in the morning and returns with that same truck and (maybe) another trailer in the afternoon. We call such a one-day home-awayhome truck tour a depot commute. The full truckload stays on its trailer, and the trailer is passed on from truck to truck until it arrives at its destination. For example: Instead of two full truckload transportations, one from Hamburg to Munich and one from Munich to Hamburg that take a whole day one-way one could exchange the trailers in Kassel to get home on the same day.

Of course, dispatching such a cooperative transportation system is a complicated task. Usually, not all transport routes will fit together. For example, if some transport traverses an edge that is not traversed in the opposite direction by any other tranport route on the same day, then this transport cannot be carried out by a sequence of depot commutes.

The organizational task to maximize the number of transports that can be operated by the CTN for Fixed Routing (FR) (i.e., with given fixed scheduled routes for all transport requests) was algorithmically studied for the first time in [2]. Let us call this problem the fixed-route CTN relay problem (FR-CTNRP). In order to evaluate the actual benefit, also monetary consequences must be assessed. Therefore, cost calculations have been provided in [3]. Using an ad-hoc optimization algorithm, it was observed in [2] that in many real-world cases the fraction of transport requests that can be cooperatively processed is too small to convince small companies to participate in a CTN. This problem was later confirmed by exact optimization calculations carried out by the author. Thus, the idea of injecting more flexibility into the problem setting was born. The first 
attempt in this direction is Multi Routing (MR), i.e., to allow for multiple routing alternatives for each transport request. The resulting problem is called the multiroute CTN relay problem (MR-CTNRP). Detailed information concerning the business process and the data handling can be read in two other contributions to this volume $[4,5]$ and the thesis $[6]$.

This paper contributes a Rapid Mathematical Programming Approach in the spirit of [7] based on mixed-integer linear programming (MILP). Based on the presented numerical results on real-world data some insights for the algorithm designer and for the manager are provided.

\section{Formal Problem Definition}

Let the set of time slots be $T=\{0,1, \ldots N\}$, where one time slot extends to half the duration of a shift. Morning time slots are even, afternoon time slots are odd $t$ 's. Any driver can drive for the length of two time slots per day, first a morning time slot, then an afternoon time slot. Let $G=(V, E)$ be the transportation network of the CTN. Its nodes $V$ are the possible trailer exchange points including all the home depots of the trucks. Its edges $E$ connect two nodes whenever it takes no more than one time slot to go from one node to the other. Each truck belongs to a depot. The set of depots is denoted by $D \subseteq V$.

Moreover, there is an index set of transport requests $Q$. For each $q \in Q$ we have an index set of routing alternatives $P_{q}$ with a default route $p(q) \in$ $P_{q}$. We set $P=\bigcup_{q \in Q}\left(\{q\} \times P_{q}\right)$. The routing alternatives are specified by a routing and scheduling function $r: P_{q} \rightarrow 2^{T \times E}$ that assigns to each routing alternative $p \in P_{q}$ for transport request $q$ a scheduled route, i.e., a set of timestamped, chronologically adjacent edges that specify when and where the trailer loaded with transport request $q$ shall go from one node to another. Origin and destination of a transport request $q$ can be read off the common origin and destination nodes of its routing alternatives. We define a depot commute as a pair of scheduled edges $(p, t, d, b)$ and $\left(p^{\prime}, t+1, b, d\right)$ where $p, p^{\prime}$ are routing alternatives, $t$ is a morning time slot, $d$ is a depot, and $b$ is some node.

For some subset of routing alternatives $P^{\prime} \subseteq P$ let $\mathcal{L}\left(P^{\prime}\right)=\bigcup_{p \in P^{\prime}}(\{p\} \times r(p))$ be the link collection of $P^{\prime}$, i.e., the total set of route-labeled scheduled transport links traversed by the routes in $P^{\prime}$. Each route $p \in P$ induces a network transport cost $\beta_{p}$ if $p \in P^{\prime}$ and a direct transport cost $\gamma_{p}>\beta_{p}$ if $p \in P \backslash P^{\prime}$. The network transport cost is calculated assuming that the transport can be carried out along the route by depot commutes of trucks only, passing on the trailer at the end of a morning time slot. The direct transport cost is calculated assuming that the transport is carried out along the route by a single driver and a single truck keeping the same trailer throughout.

The task of the MR-CTNRP is to find a (by some criterion) optimal subset $P^{*} \subseteq P$ whose link collection $\mathcal{L}\left(P^{*}\right)$ can be partitioned into loaded depot commutes and for which at most one routing alternative is chosen for each transport request. For an MR-CTNRP-solution $P^{*}$, nettrips are the transport requests using a route from $P^{*}$ cooperatively, and dirtrips are remaining transport requests 
using the default route directly. The FR-CTNRP is the special case of the MRCTNRP where $\left|P_{q}\right|=1$ for all $q \in Q$, i.e., there is only one routing alternative for each transport request.

The optimality criterion we investigated for this work are, first, the maximization of the number $\left|P^{*}\right|$ of nettrips and, second, the minimization of the total transportation cost incurred by nettrips and dirtrips.

\section{A Mixed-Integer Linear Programming Model}

We use the principle of "Rapid Mathematical Programming", first systematically discussed in [7]. Moreover, we use the modeling language zimpl introduced in the same work. Our goal is to provide a lean model for the MR-CTNRP with enough flexibility to evaluate model variants fast. We define binary selection variables $z_{p}$ for each routing alternative $p \in P$. Moreover, we define binary depot assignment variables $x_{p, t, d, b}$ and $x_{p, t, a, d}$ indicating that $p$ uses the departure link $(d, b)$ or home link $(a, d)$, respectively, of a depot commute at time slot $t$. We introduce additional non-negative measurement variables for the number of nettrips $(u$ integer) and the total cost ( $v$ continuous) in order to be able to read off those values from the solution values. The resulting model reads as follows:

$$
\max u \text { or } \min v
$$

such that

$$
\begin{aligned}
& u-\sum_{p \in P} z_{p}=0 \\
& v-\sum_{p \in P} \beta_{p} z_{p}-\sum_{q \in Q} \gamma_{p(q)}\left(1-\sum_{p \in P_{q}} z_{p}\right)=0 \\
& x_{p, t, d, b}-z_{p}=0 \quad \forall(p, t, d, b) \in \mathcal{L}(P) \\
& \text { with } d \in D, t \text { even }(4) \\
& x_{p, t, a, d}-z_{p}=0 \quad \forall(p, t, a, d) \in \mathcal{L}(P) \\
& \text { with } d \in D, t \text { odd (5) } \\
& \sum_{\substack{p \in P: \\
(p, t, d, b) \in \mathcal{L}(P)}} x_{p, t, d, b}-\sum_{\substack{p \in P: \\
(p, t+1, b, d) \in \mathcal{L}(P)}} x_{p, t+1, b, d}=0 \quad \forall d \in D, \forall t \in T \text { even }(6) \\
& \sum_{\substack{p \in P: \\
(p, t, a, d) \in \mathcal{L}(P)}} x_{p, t, a, d}-\sum_{\substack{p \in P: \\
(p, t-1, d, a) \in \mathcal{L}(P)}} x_{p, t-1, d, a}=0 \quad \forall d \in D, \forall t \in T \text { odd }(7) \\
& \sum_{p \in P_{q}} z_{p} \leq 1 \quad \forall q \in Q \\
& x_{p, t, d, b}, z_{p} \in\{0,1\} \forall(p, t, d, b) \in \mathcal{L}(P) .
\end{aligned}
$$

We optimize in the objective function (1) one of the measurement variables $u$ or $v$. Restrictions (2) and (3) compute these values from the independent 
variables. Restrictions (4) and (5) ensure that a path can be selected if and only all of its morning links and all of its afternoon links have been assigned to the appropriate part of some depot commute. Restrictions (6) and (7) guarantee that for each depot commute the number of paths assigned to the leaving part of the commute equals the number of paths assigned to the returning part. In restriction (8) we allow for at most one selected route per transport request. Whenever we want to compare the FR optimum, we can fix the selection variables to be one on the fixed route only. This way, we can easily test variants of possible CTN operations with only slight modifications.

\section{Computational Results}

In Table 1 we show the results of our tests. In numerical experiments we computed optimal solutions for model variants on the basis of real-world instances. The experiments with 6 start time slots ( $\mathrm{t} 6$ ) are typical instance sizes for a daily operation on the moving horizon. The experiments with 14 start time slots (t14) have been carried out in order to evaluate the value of the additional future information. We applied two strategies to keep the number of routes under control: The instances 13 allow only routes with at most 3 nodes, the instances 15 allow routes with at most five notes. Moreover, we distinguish the case in which shifting is allowed: shifting (-s) provides for each single-link transport request a canonical routing alternative that uses the same single-link route but on the other time slot of the same day. For each solution we are interested in the fraction of transport requests that are carried out cooperatively and in the cost savings incurred by cooperative transportation. The instances maxtrips maximize the number of nettrips, the instances mincost minimize total costs.

All instances were provided by Bernd Nieberding from the ILAN project at the FH Erfurt. The cost for each route and transportation mode was estimated by the ILAN project team according to [3].

We used zimpl 3.3.1/cplex 12.5.0.0 on a MacBookPro (2012)/MacOS 10.11.6. The cplex parameter were changed to "set timelimit 3600" (timeout), "set mip tol mipgap 0.01" (increased optimality gap), "set mip tol integrality 1e-7" (decreased integrality gap), and "set emph mip 1" (search preferably for integer feasible solutions).

The following insights can be drawn from Table 1:

1. Our model scales well for alternative routes of length at most three nodes; the computation times explode if the maximal path length is increased to five nodes. Managerial insight: stick with routes of length at most three. Algorithmic insight: if longer routes need to be handled in the model for some reason, then more sophisticated solution methods are needed (most notably dynamic column generation for alternative routes). Possible explanation: with routes of at most three nodes (starting in the morning and arriving in the afternoon), our model reveals that then all decisions can be made independently for each day, so that the solution method scales well with an increasing number of time slots. 


\begin{tabular}{|c|c|c|c|c|c|c|c|c|}
\hline$\overbrace{0}^{2}$ & 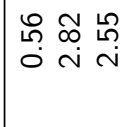 & 怘 웅 & 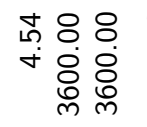 & 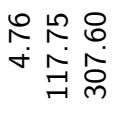 & 合兽奇 & 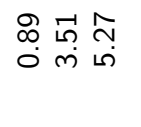 & 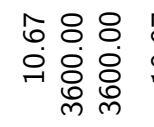 & 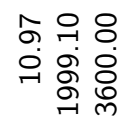 \\
\hline & 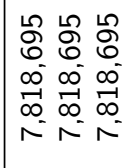 & 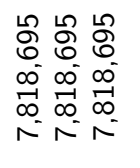 & 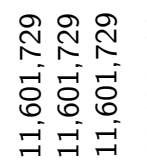 & 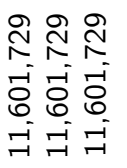 & 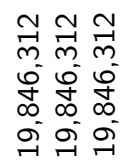 & 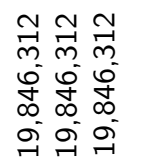 & 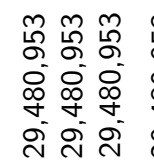 & 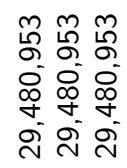 \\
\hline & 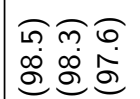 & 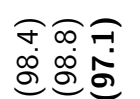 & 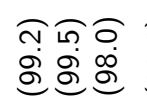 & 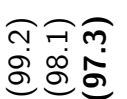 & & & 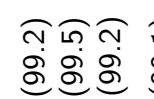 & 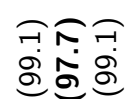 \\
\hline & 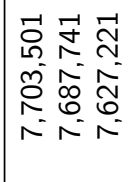 & 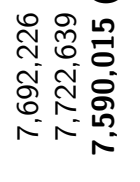 & $\begin{array}{l}\llcorner 00 \\
6 \\
6\end{array}$ & 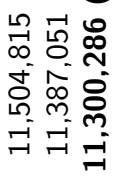 & 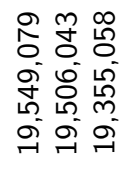 & 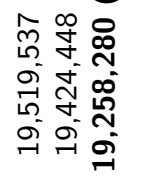 & 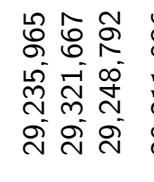 & 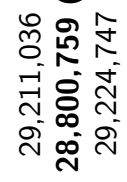 \\
\hline & 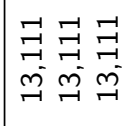 & 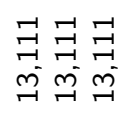 & 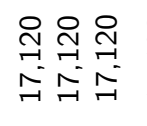 & 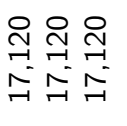 & 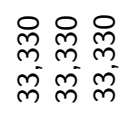 & 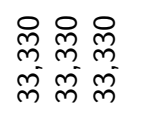 & 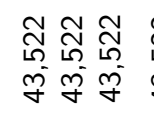 & 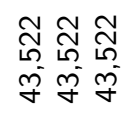 \\
\hline & 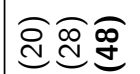 & 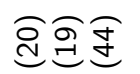 & ્ㅗㅀ & త્త્વ & 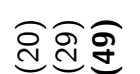 & 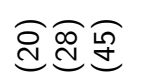 & 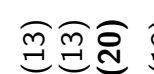 & 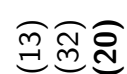 \\
\hline & 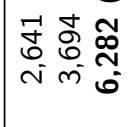 & 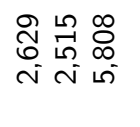 & 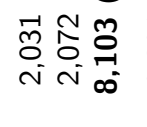 & 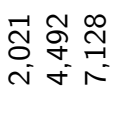 & 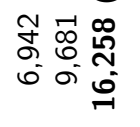 & & 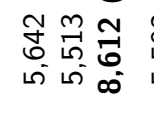 & 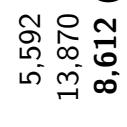 \\
\hline & 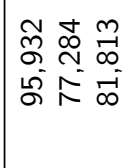 & 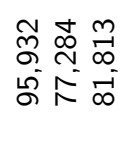 & 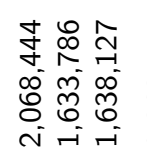 & 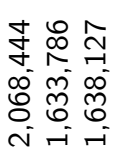 & 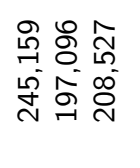 & 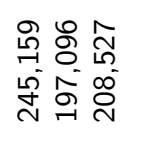 & 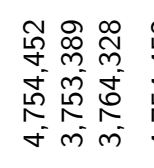 & \\
\hline & 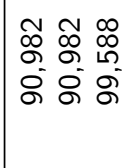 & 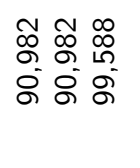 & 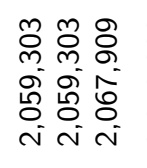 & 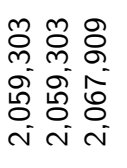 & 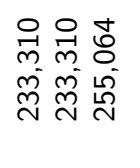 & 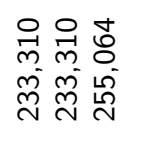 & 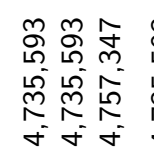 & \\
\hline 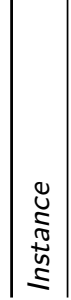 & 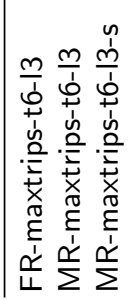 & 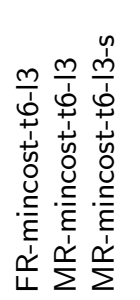 & 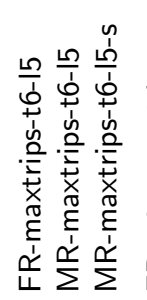 & 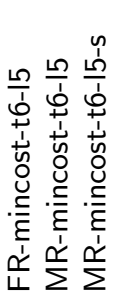 & 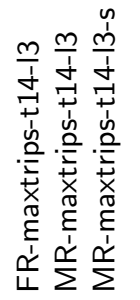 & 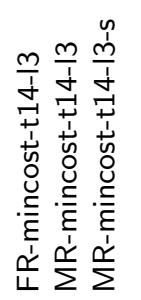 & 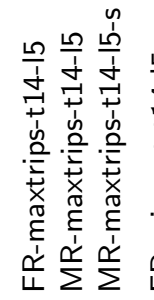 & 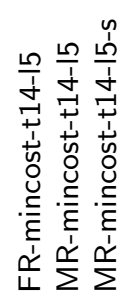 \\
\hline
\end{tabular}

Table 1. Computational results: FR/MR: fixed route/multiple routes; maxtrips/mincost: maximize network trips/minimize costs; $\mathrm{t} 6 / \mathrm{t} 14$ : start at latest at time slot $6 / 14$; 13/15: routes have at most $3 / 5$ nodes; vars: no. of model variables; cons: no. of model constraints; nettrips: no. of transport requests operated cooperatively; dirtrips: total no. of transport requests; netcost: total cost for solution; dircost: total cost for individual transportation only; CPU/s: runtime with timeout set to 3600.00 
2. The idea to provide multiple routes for each transport request is effective; the optimal number of nettrips increases by around $50 \%$. Managerial insight: motivate companies to provide alternative routes.

3 . The idea to allow of shifting is very effective: it roughly yields another $60 \%-$ $70 \%$ increase of possible nettrips. Managerial insight: utilize both time slots of each day for single-link transports.

4. Maximizing nettrips usually yields low-cost solutions, and minimizing costs usually yields high-nettrips solutions. Managerial insight: use a suitable combination of both, e.g., if driver satisfaction is important in its own right.

5. Cost minimization seems to scale better in the MILP solution process with problem size (see MR-mincost-t14-15, which yield a better fraction of nettrips than both MR-maxtrips-t14-15 and MR-maxtrips-t14-15-s). Algorithmic insight: for maximizing nettrips, perturb the objective by a cost term.

6. A longer horizon does not lead to a substantially larger fraction of nettrips. Managerial insight: the model is suitable for a rolling-horizon planning on a short planning horizon. Possible explanation: again, the model for short paths is uncoupled over days.

The rapid mathematical programming investigation with exact mathematical software yields useful information for the design and operation of a CTN. Even more (capacities, fair share of benefit, etc.) could be incorporated. We showed that almost half of the transports can be carried out as nettrips in a CTN with multirouting and shifting. Lifting additional potential of the CTN concept requires a more sophisticated machinery of Mathematical Programming - which lifts the entry hurdle for small companies maybe too high. Thus, there is evidence for the fact that multirouting plus shifting on single-day routes (length 3) yields a well-balanced CTN operation mode with high potential.

\section{References}

1. Apfelstädt, A., Gather, M.: New design of a truck load network. In: Dynamics in Logistics: Proceedings of the 4th International Conference LDIC, 2014 Bremen, Germany. Lecture Notes in Logistics, Springer International Publishing (2014) 183191

2. Apfelstädt, A., Dashkovskiy, S., Nieberding, B.: Modeling, optimization and solving strategies for matching problems in cooperative full truckload networks. IFACPapersOnLine 49 (2016) 18-23

3. Dashkovskiy, S.N., Nieberding, B.: Costs and travel times of cooperative networks in full truck load logistics. In: Dynamics in Logistics. Springer International Publishing (2016) 193-201

4. Apfelstädt, A.: New production approach for european truckload cargo industry. In: This volume. (2017)

5. Nieberding, B.: Multi-routing for transport-matching in cooperative, full truckload, relay networks. In: This volume. (2017)

6. Apfelstädt, A.: Handlungsoptionen im euronationalen Ladungsverkehr. Dissertation, Bergische Universität Wuppertal (2017) Cuvillier, ISBN 9783736995635.

7. Koch, T.: Rapid Mathematical Programming. Dissertation, Technische Universität Berlin (2004) 